\title{
2016 Decadal Update of the NASA ESTO Lidar Technologies Investment Strategy
}

\author{
Azita Valinia ${ }^{1}$, David M. Tratt ${ }^{2}$, William T. Lotshaw ${ }^{2}$, and Kevin M. Gaab ${ }^{2}$, George J. Komar ${ }^{1}$ \\ ${ }^{1}$ NASA Earth Science Technology Office, 8800 Greenbelt Road, Greenbelt, MD 20771, USA \\ ${ }^{2}$ The Aerospace Corporation, P.O. Box 92957, Los Angeles, CA 90009-2957, USA \\ Authore-mail address: Azita.Valinia-1@nasa.gov
}

\begin{abstract}
We describe the 2016 update of the NASA Earth Science Technology Office (ESTO) investment strategy in the area of lidar technologies as pertaining to NASA's Earth Science measurement goals in the next decade.

OCIS codes: (010.3640) Lidar; (140.0140) Lasers and laser optics; (040.0040) Detectors.
\end{abstract}

\section{Introduction}

This study summarizes the 2016 state-of-the-art in lidar technology as it pertains to Earth science and discusses needed capabilities for achieving NASA's Earth science measurement goals. It updates the last Earth Science Technology Office (ESTO) lidar investment strategy, documented a decade ago [1], which laid out the scientific basis and key technology developments needed to achieve NASA's Earth science goals.

The overall space-based lidar applications landscape is such that comparatively little of the total technology trade space can claim actual space heritage, leaving considerable scope for further development and exploitation. However, the analogous situation for airborne ("suborbital" in NASA parlance) lidar indicates that a substantial reservoir of experience and heritage is available in that domain to draw on for seeding future development of spacebased implementations.

In the realm of lidar-specific technologies, progress in the last decade has been mixed. Some emerging laser materials (e.g., Cr:ZnSe) and improvements in nonlinear optical (NLO) materials have expanded options for wavelength generation both in the near-UV and shortwave-to-midwave infrared (SWIR-MWIR), while dramatic improvements in pump laser-diode electrical efficiency have significantly improved the wall-plug efficiency (WPE) of both bulk solid-state and fiber-based lasers.

\section{General observations}

An especially important consequence of laser technology development over the past decade is that fiber-laser average power capability now rivals that of traditional bulk solid-state systems, which is a distinct advantage in that all-fiber architectures are both compact, immune to misalignment, and typically exhibit higher WPE than conventional bulk solid-state lasers. The significance of these developments is that previously the restricted performance envelope of fiber-based lasers had ruled them out of consideration for measurement applications requiring high average power or moderate (i.e., $\sim \mathrm{mJ}$ ) pulse energy. Developments of the past decade merit a reevaluation in some of these scenarios. For instance, high pulse repetition frequency (PRF) transmitters have generally been regarded as inconsistent with the typical laser altimetry CONOPS (Concept of Operations) because of a perceived tendency for ranging ambiguity when there are multiple pulses in contention. However, the waveform agility of telecomm-heritage signal laser diodes enables novel temporal waveforms that would permit disambiguation of range/altimetry measurements in such cases, and moreover would do so with the corollary SWaP (size, weight, and power) benefits of fiber lasers, as well as the additional advantages named above.

While the relief offered to laser performance requirements by improved detectors has long been recognized, technological investment has remained heavily biased toward laser development. A consistent theme expressed across all measurement scenarios was the need for improved detector performance, particularly radiation-hardened multi-element architectures with high quantum efficiency, low noise, low timing jitter, and low afterpulsing. Improved materials growth and device fabrication and processing techniques, particularly for complex bandengineered materials, could increase detector yield as well as improve device dark count rates, afterpulsing performance, and non-uniformity, which will be necessary for the new generation of array detectors. The astronomy and astrophysics communities pursue major detector development programs that could also be leveraged in this regard. A summary of scientific measurements and associated technology needs (without prioritization of technology development) is shown in Figure 1.

Of the laser technologies covered in the 2006 report and this update, solid-state systems operating at $1 \mu \mathrm{m}$ and lidars using harmonics thereof are by far the most mature and closest to insertion once the arduous task of space 
qualification is accomplished. The laser technologies required for direct detection UV Doppler wind measurements, and NIR/Vis/UV atmospheric aerosol/cloud/ecosystem measurements, have one remaining obstacle which should be within reach: improving the damage resistance and reliability of harmonic generation components. This task principally requires improved contamination control and more robust anti- and high-reflection coatings at UV wavelengths. Fiber-laser and fiber/bulk solid-state analogs of high-PRF, moderate energy $(\leq 1 \mathrm{~mJ})$ bulk solid-state systems are also rapidly approaching the critical TRL6 benchmark and could displace the bulk solid-state technology entirely in a few years. It is instructive to note that the NFIRE (Near-Field InfraRed Experiment) spacebased lasercomm experiment has been operational for 9 years, using relatively primitive fiber amplifier components.

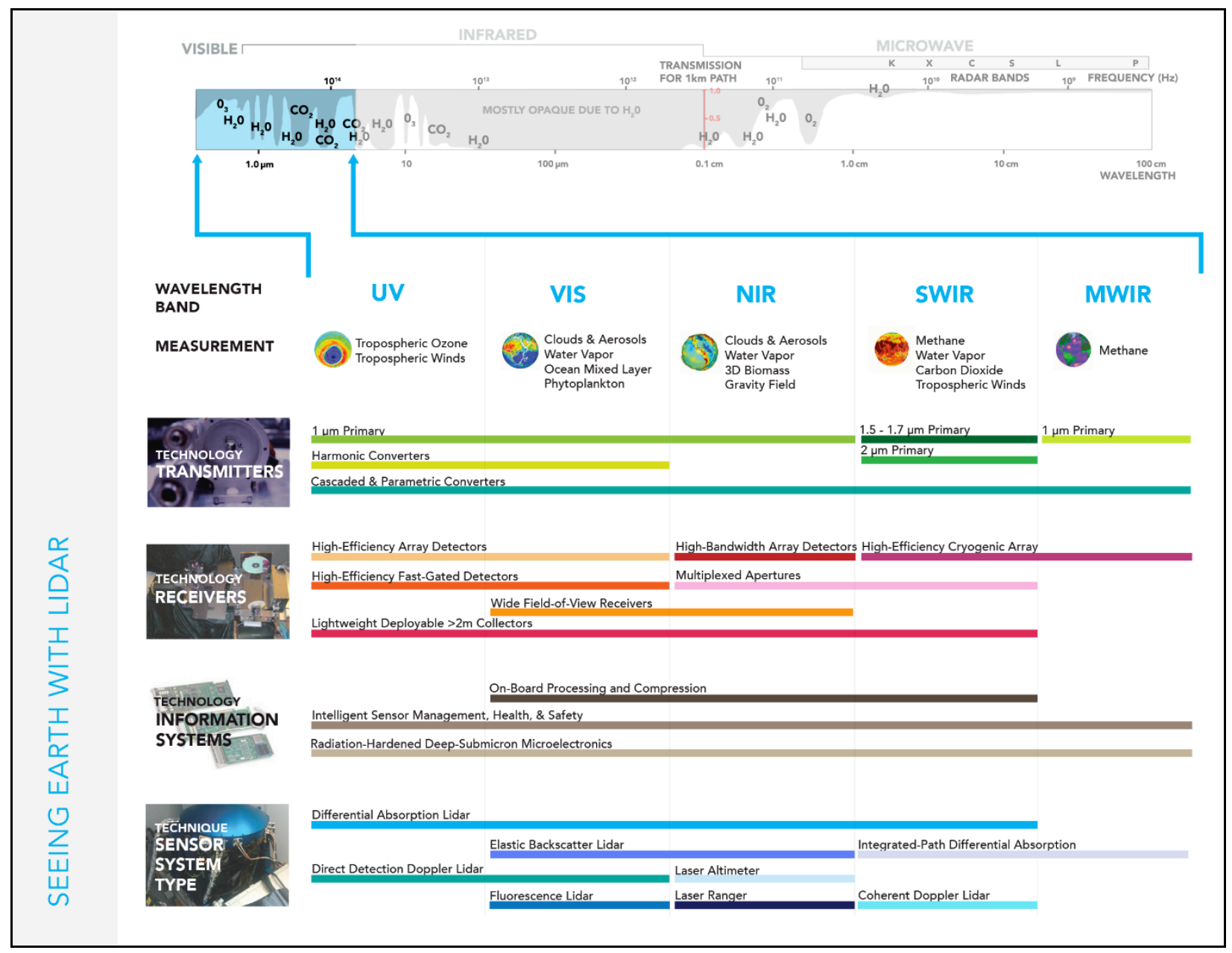

Figure 1. Summary of Earth science lidar applications and associated top-level technology needs.

As the analysis progressed it became evident that a number of technology areas identified as important in the 2006 report [1] had not advanced significantly in the ensuing decade and on that basis are being carried forward into the current report. Based on community inputs to this 2016 update [2; Appendix 3A], 1570-nm sources for ASCENDS, 2- $\mu \mathrm{m}$ sources for coherent winds, and even 1064/532/355-nm sources for aerosol/clouds/ecosystem measurements appear to have shown little TRL advancement when compared to the equivalent entries in the 2006 report [1; Appendix 5A]. The technology enterprise should consider re-prioritization of these efforts once the current Decadal Survey panel has communicated its recommended science priorities. For this reason, the current update avoids assignment of priorities to either the measurement objectives listed or the associated technology requirements.

\section{Recommendations}

It is important to note that the 2016 survey of lidar technologies was conducted against the background of a radical retooling of the space sensing arena occasioned by an explosion of SmallSat and/or hosted payload concepts that have evolved in response to an increasingly cost-constrained environment. SmallSats, especially the U-class 
concepts currently in vogue, demand a greater degree of attention to miniaturization and efficiency than has heretofore been necessary. Hence, in addition to the conventional lidar technologies that dominated the 2006 survey, the current analysis also advocates a number of technologies such as integrated photonic circuitry and deepsubmicron microelectronic architectures that, while non-lidar specific, nevertheless offer considerable advantages (in some cases enabling) to lidar measurement concepts intended to be compatible with SmallSat/U-class buses. In this respect, the emergence of innovative manufacturing techniques in the last decade offers pathways to the creation of, for instance, structural elements and large-area reflectors that are lightweighted in ways not feasible through conventional means.

It is the recommendation of the report that the Earth science technology portfolio be actively partitioned to create a more symbiotic balance between emerging technologies and the ongoing development of more mature technologies. Emerging technologies such as integrated photonics, high-PRF fiber lasers, array detectors, and tunable NLO (nonlinear optical) schemes are some areas that might be leveraged under such a redirected program.

It is further recommended that provisions for classified technology development proposal appendices be instituted, analogous to the policy implemented by the NASA Earth Venture program. This would provide a mechanism whereby technological advances developed by the national security community could be leveraged by proposers in an environment where ESTO could be assured that the technologies in question are truly viable.

Several critical technology areas (notably NLO materials and associated optical coatings, and also detectors) continue to suffer from a significant shortfall in domestic capability, with many system and component technologies being dominated by non-U.S. vendors. This presents challenges to U.S.-based mission planners due to the prohibition on explicitly advising or guiding foreign technology development imposed by ITAR/EAR regulations. It is therefore recommended that these areas of need be brought to the attention of the Committee on National Security Systems (CNSS) Supply Chain Risk Management (SCRM). At the time of this writing, the SCRM was engaged in reviewing changes to CNSS 505 in order to address the full spectrum of SCRM policy across the entire U.S. Government. The updated document was scheduled for release in the April/May 2016 timeframe.

Finally, systems engineering should be more effectively employed as an arbitrator between evolving technology options, by enabling parametric trades between aperture size, detector efficiency, laser power, waveform diversity, etc. that could circumvent technological hurdles. To be successful, this approach requires robust, high-fidelity modeling and simulation capabilities, in both the environmental and sensor performance domains, which will require strengthening and further development of concurrent engineering tools. A significant body of knowledge relating to model-based system engineering (MBSE) exists within the defense community, where specialized analyses are routinely conducted within a generalized MBSE construct.

The MBSE paradigm offers an approach for independently arbitrating a number of questions prevailing within the lidar community. For example, three decades of effort have thus far failed to produce a viable solution for a perennial high-priority measurement, namely 3-D tropospheric winds. This particular question has been so resistant to resolution that instead of converging on an optimum approach the number of candidate options has instead expanded in recent years. The search for a technological pathway to the ASCENDS (Active Sensing of $\mathrm{CO}_{2}$ Emissions over Nights, Days, and Seasons) mission currently threatens to follow a similar trajectory, with at least three options under concurrent investigation and an absence of concerted pressure to converge on an optimal approach. Another question that could be addressed by a rigorous MBSE analysis would be whether the global topography mission is most efficiently mounted using a single platform or a distributed architecture involving SmallSat constellations. Each of the analyses identified above would help to resolve long-running uncertainties, enabling NASA to more effectively target available resources.

\section{Acknowledgements}

In the preparation of the 2016 report, ESTO actively solicited and received input from the community. Written inputs by the community were submitted through ESTO's white paper input site. ESTO also organized three workshops with subject matter experts at NASA Goddard Space Flight Center, NASA Langley Research Center, and NASA Jet Propulsion Laboratory. ESTO also convened a virtual community forum to gather additional input from the community at large.

\section{References}

[1] J. Neff and A. Valinia (eds.), Working group report: Lidar technologies (NASA ESTO, 2006), http://esto.nasa.gov/files/LWGReport2006.pdf.

[2] A. Valinia, D. Tratt, and D. Mayo (eds.), 2016 NASA Earth Science Technology Office (ESTO) lidar technologies review (NASA ESTO, 2016), http://esto.nasa.gov/LidarStrategies/Lidar_TechStrategy_\%202016.pdf. 